Check for updates

Cite this: J. Mater. Chem. A, 2021, 9, 14691

Received 22nd March 2021 Accepted 4th June 2021

DOI: $10.1039 / \mathrm{d} 1 \mathrm{ta0} 2399 \mathrm{c}$

rsc.li/materials-a

\section{Design of triple and quadruple phase boundaries and chemistries for environmental $\mathrm{SO}_{2}$ electrochemical sensing $\dagger$}

\begin{abstract}
Air pollution is a major environmental risk to the health of humans and vegetation, leading to increasing demand for real-time monitoring of toxins and harmful pollutants. Recently, the first type III electrochemical sensors tracking lethal concentrations of $\mathrm{SO}_{2}$ were developed based on a fast-conducting $\mathrm{Li}$ garnet $\mathrm{Li}_{7} \mathrm{La}_{3} \mathrm{Zr}_{2} \mathrm{O}_{12}$ (LLZO) solid electrolyte. Despite the successful proof-of-concept demonstration, the ideal sensing electrode issues concerning its preferred microstructure and chemistry remain unresolved as no catalysts are involved to ensure the low cost of such future devices. The challenging task to secure an efficient ionic, electronic, and gas pathways are needed for further sensor performance improvement. Here, the focus was placed on manipulating the electrochemical reaction zones from triple phase boundaries (TPBs) to quadrupole phase boundaries (QPBs) by merely changing the processing temperature of a $\mathrm{Li}_{2} \mathrm{SO}_{4}-\mathrm{CaSO}_{4}$ composite sensing electrode. The intended manipulation enforced the desired shift from TPB to QPB reaction zones and unlocked a larger effective surface area for the electrochemical reaction. The sensor operated at $480{ }^{\circ} \mathrm{C}$ with up to oneorder-of-magnitude-lowered response time and up to a $75 \%$ decrease in the recovery time down to $\sim 5 \mathrm{~min}$ for the QPB-based configuration compared to the TPB-based one. This study demonstrates novel tools and strategies to favorably engineer sensing reaction zones through electrode processing techniques and enrich the functionality of the Ligarnet $\mathrm{Li}_{7} \mathrm{La}_{3} \mathrm{Zr}_{2} \mathrm{O}_{12}$ fast-conducting electrolyte for sensing applications beyond batteries.
\end{abstract}

\section{Introduction}

The highly reactive sulfur dioxide $\left(\mathrm{SO}_{2}\right)$ gas negatively contributes to particle pollution, visibility (haze), health, and climate

${ }^{a}$ Department of Materials Science and Engineering, Massachusetts Institute of Technology, 77 Massachusetts Avenue, Cambridge, MA 02139, USA. E-mail: jrupp@ mit.edu

${ }^{b}$ Department of Electrical Engineering and Computer Science, Massachusetts Institute of Technology, 77 Massachusetts Avenue, Cambridge, MA 02139, USA

$\dagger$ Electronic supplementary information (ESI) available. See DOI: $10.1039 / \mathrm{d} 1 \mathrm{ta} 02399 \mathrm{c}$ via its oxidation to sulfuric acid and reaction with other compounds in the atmosphere. The main sources of $\mathrm{SO}_{2}$ emissions are the combustion of sulfur-containing materials (e.g., coal and oil resources) at power plants and in the oil and gas industry, ${ }^{1}$ which are typically accompanied by the release of greenhouse gases into the atmosphere and acid rain. Additional sources of $\mathrm{SO}_{2}$ emissions include degassing volcanic sources and ore smelters. Exposure to $\mathrm{SO}_{2}$ and subsequent particulate matter (e.g., sulfates) can lead to severe health problems, including breathing difficulties, respiratory illnesses, and heart and lung diseases, and is life-threatening once concentrations above $100 \mathrm{ppm}$ are reached. Although the air pollution issue is globalizing, recent trends in global $\mathrm{SO}_{2}$ emissions show that developing countries are the predominant world-wide emission contributors, with a share of $83 \% .^{2}$ Therefore, novel costeffective, continuous, and real-time tracking technologies are urgently needed to further reduce $\mathrm{SO}_{2}$ emissions, especially in developing nations.

Currently, commercial analytical tools based on infrared (IR) and ultraviolet (UV) optical technologies can accurately detect

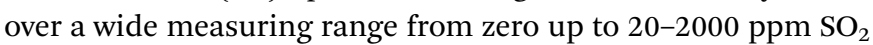
at high resolution ( $1 \mathrm{ppm}$ to $1 \mathrm{ppb}$ ) and with a fast response of 10-60 s. ${ }^{3-5}$ Nonetheless, such instruments suffer from a large power demand and high cost, are rather bulky in size, and are not necessarily suited for real-time monitoring, which limit their active use-cases. In the quest for continuous monitoring of $\mathrm{SO}_{2}$ pollutants, portable and smaller-sized solid-state electrochemical sensors represent a promising alterative to UV and IR optical sensors.

Among the plethora of solid-state electrochemical sensors, the so-called potentiometric sensors operating under thermodynamic equilibrium are a promising technology based on the conversion of chemical quantities into electrical signals, namely an open-circuit voltage, according to the Nernst equation (see the schematic in Fig. 1). Owing to their simple structure and operating principles, potentiometric electrochemical sensors can track $\mathrm{SO}_{2}$ gases by coupling an auxiliary sensing electrode (SE) to an ion-conducting solid electrolyte and 

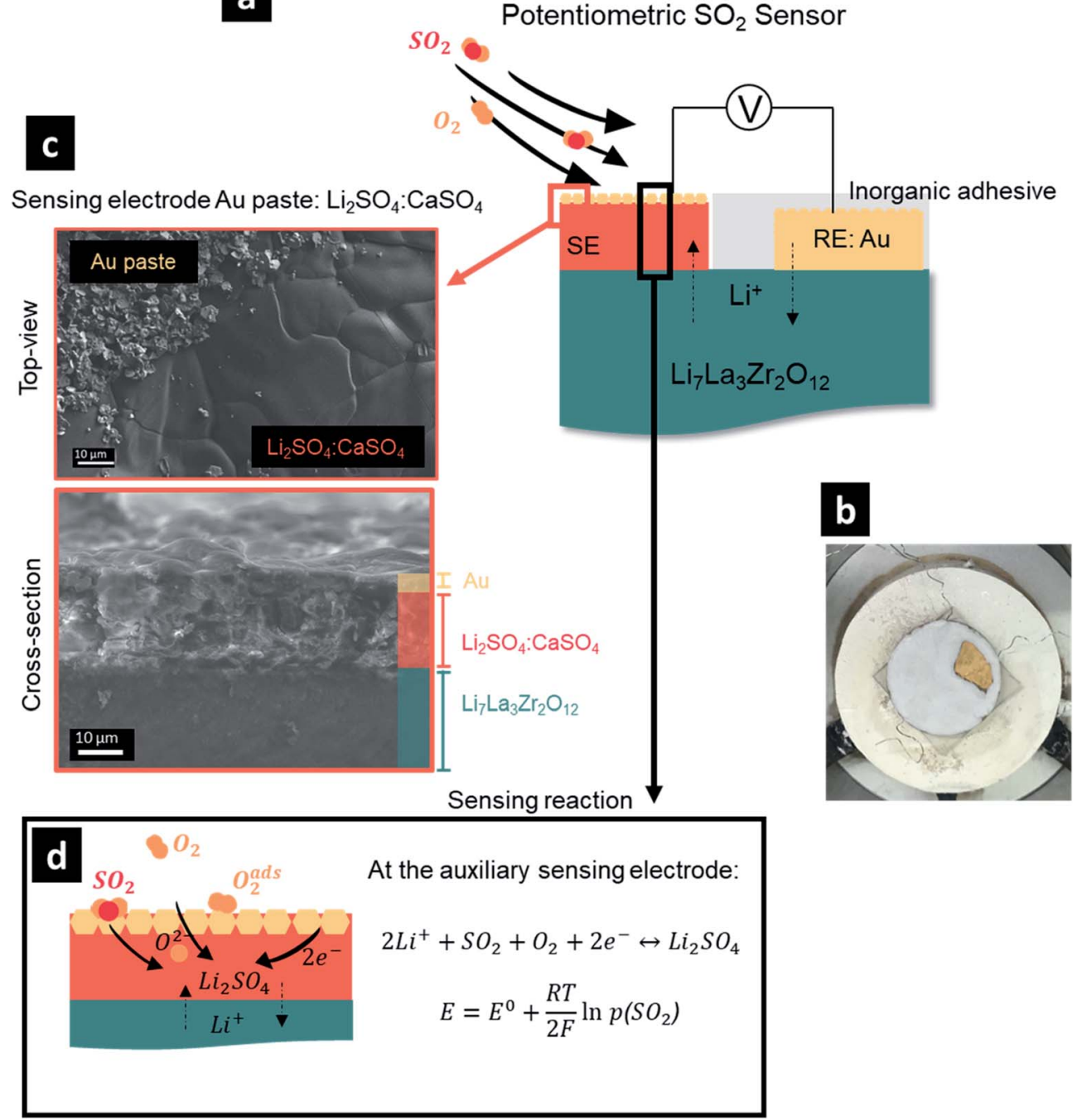

Fig. 1 (a) Schematic illustration of an electrochemical potentiometric $\mathrm{SO}_{2}$ sensor based on a $\mathrm{Li}_{7} \mathrm{La}_{3} \mathrm{Zr}_{2} \mathrm{O}_{12}$ solid electrolyte, gold reference electrode, and $\mathrm{Li}_{2} \mathrm{SO}_{4}: \mathrm{CaSO}_{4}$ sensing electrode in an in-plane design. (b) Pellet-scale $\mathrm{SO}_{2}$ sensor (all covered with inorganic adhesive except the sensing electrode) in an environmental chamber during electrochemical testing connected with platinum wires. (c) Top-view (top) and cross-sectional (bottom) SEM micrographs of the sensing electrode on top of the $\mathrm{Li}_{7} \mathrm{La}_{3} \mathrm{Zr}_{2} \mathrm{O}_{12}$ solid electrolyte as a part of the sensor cell structure. (d) Schematic illustration of the reaction mechanism occurring at the sensing electrode including diffusion of gaseous $\mathrm{O}_{2}$ and $\mathrm{SO}_{2}$, adsorption of $\mathrm{O}_{2}$ at the surface of the sensing electrode, diffusion of adsorbed oxygen toward reaction sites, and transfer of electrons to form adsorbed $\mathrm{O}^{2-}$, accompanied by the diffusion of $\mathrm{Li}^{+}$ions to form $\mathrm{Li}_{2} \mathrm{SO}_{4}$. The emf of the cell is determined according to the Nernst equation and is only a function of the partial pressure of $\mathrm{SO}_{2}$ (considering fixed temperature and partial pressure of $\mathrm{O}_{2}$ ).

a reference electrode (RE) (Fig. 1). The most widely used solidelectrolyte material groups for monitoring $\mathrm{SO}_{2}$ are oxygen-ion $\left(\mathrm{O}^{2-}\right)$ conductors such as yttria-stabilized zirconia ${ }^{6}$ and magnesia-stabilized zirconia, ${ }^{7}$ and $\mathrm{Na}^{+}$-ion conductors such as sodium beta-alumina ${ }^{8}$ or NASICON. ${ }^{9}$ Despite the proof-ofconcept demonstration of their ability to fulfill the role as an electrolyte for these sensors, their rather low carrier mobility typically necessitates high operating temperatures beyond $500{ }^{\circ} \mathrm{C}$ to ensure sufficient ionic conductivity and a fast response and recovery time for the sensor. It has been shown that developments in solid-state batteries $(\mathrm{SSBs})^{10}$ have been strongly connected to the new discoveries of novel solid-state electrolyte and electrode candidates, ${ }^{\mathbf{1 1}, 12}$ which have recently been slowly integrated in beyond-battery applications such as sensors and memristors. ${ }^{\text {13-15 }}$

Very recently, a first proof-of-concept for a potentiometric type III $\mathrm{SO}_{2}$ sensor (where the auxiliary sensing electrode contains both the gaseous $\mathrm{SO}_{2}$ species and the $\mathrm{Li}^{+}$mobile carrier of the solid electrolyte) based on a $\mathrm{Li}_{2} \mathrm{SO}_{4}-\mathrm{CaSO}_{4}-\mathrm{LLZO}$ composite sensing electrode and $\mathrm{Li}_{6.54} \mathrm{La}_{3.00} \mathrm{Zr}_{1.36} \mathrm{Ta}_{0.50} \mathrm{O}_{11.73}$ solid electrolyte was demonstrated, showing a close-to-

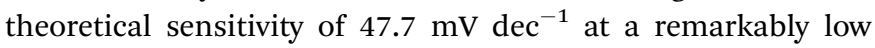
operating temperature of the sensor of $240{ }^{\circ} \mathrm{C} .{ }^{13}$ This work demonstrated the ability to widen the range to track more gas pollutants from $\mathrm{CO}_{2}$ to $\mathrm{SO}_{2}$ with a similar sensor geometry. ${ }^{\mathbf{1 3 , 1 6}}$ Nonetheless, the $\mathrm{SO}_{2}$ sensing performance was shown to be 
limited with a long response time on the order of $\sim 30-40 \mathrm{~min}$, which was attributed to the sluggish kinetics at the sensingelectrode interfaces and the rather dense microstructure of the sensing electrode. As the sensing-electrode reaction primarily occurs at the ionic/electronic/gas junctions, it was evident that creating and making interconnected designs may be an interesting pathway for the future; however, this route has not yet been tackled.

Through this work, we target the existing issues to further improve the response and recovery time of Li-garnet-based $\mathrm{SO}_{2}$ sensors by defining and controlling the quadruple-phaseboundary (QPB) reaction zones of the sensing electrode microstructure. In general, the electrochemical sensing reaction occurs at the so-called triple-phase boundary (TPB), a junction between the sensing electrode/electrolyte $/ \mathrm{SO}_{2}$ gas phase. In this case, the sensing electrode is typically processed as a dense ceramic entity on top of a dense electrolyte, and open triple points toward the gas phase are the effective reaction zones (see the schematic in Fig. 2). The performance of $\mathrm{SO}_{2}$ sensors relies on a composite electrode, such as a phase mixture of $\mathrm{Li}_{2} \mathrm{SO}_{4}-$ $\mathrm{CaSO}_{4}$, to ensure a stable and fast electrochemical reaction. One may envision that instead of a dense composite sensor electrode, an auxiliary sensing structure incorporating porosity to foster interconnected reaction zones could be used, which would newly define a quadruple-phase boundary (QPB), where the electrochemical reaction takes place between the auxiliary

a Dense sensing electrode microstructure $\left(T_{\text {processing }}=750^{\circ} \mathrm{C}\right)$

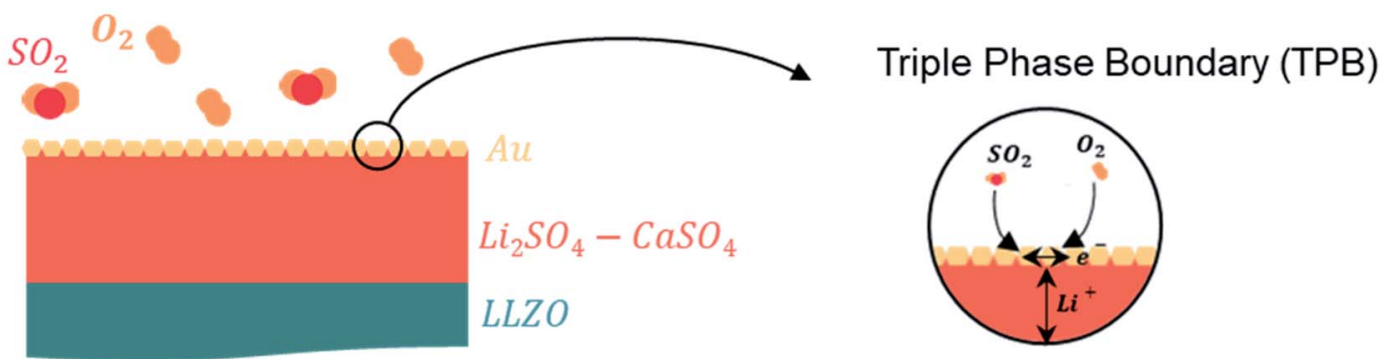

Top-view SEM: Auxiliary Sensing Electrode

Cross section SEM: Au/Li $\mathrm{SO}_{4}-\mathrm{CaSO}_{4} / \mathrm{LLZO}$
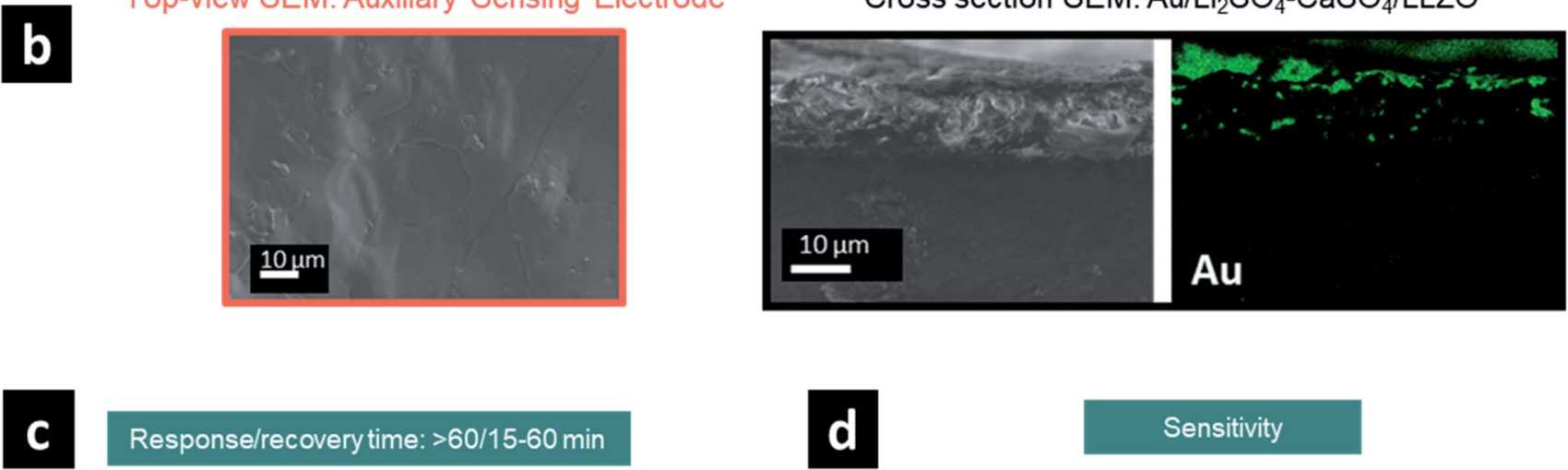

\section{Sensitivity}
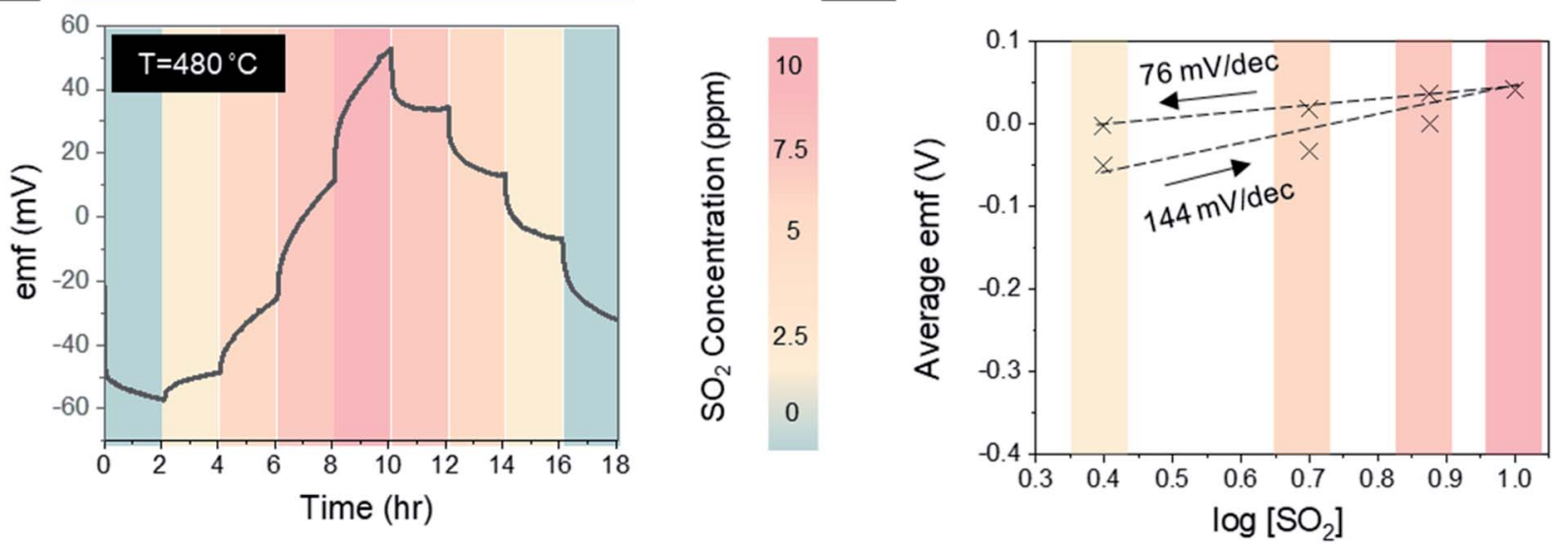

Fig. 2 (a) Schematic illustration of the $\mathrm{SO}_{2}$ sensor with a dense sensing electrode microstructure. (b) Top view and cross-sectional SEM micrographs and elemental analysis (Au) of dense auxiliary sensing electrodes processed at $750{ }^{\circ} \mathrm{C}$. (c) Emf response to $\mathrm{SO}_{2} \mathrm{Concentration}$ step change $(0-10 \mathrm{ppm})$ at $480^{\circ} \mathrm{C}$ of the dense auxiliary sensing electrodes (recovery/response times are stated above and were extracted from the graphs). (d) Emf dependence on the $\mathrm{SO}_{2}$ concentration on a logarithmic scale showing sensitivity for the dense auxiliary sensing electrodes. 

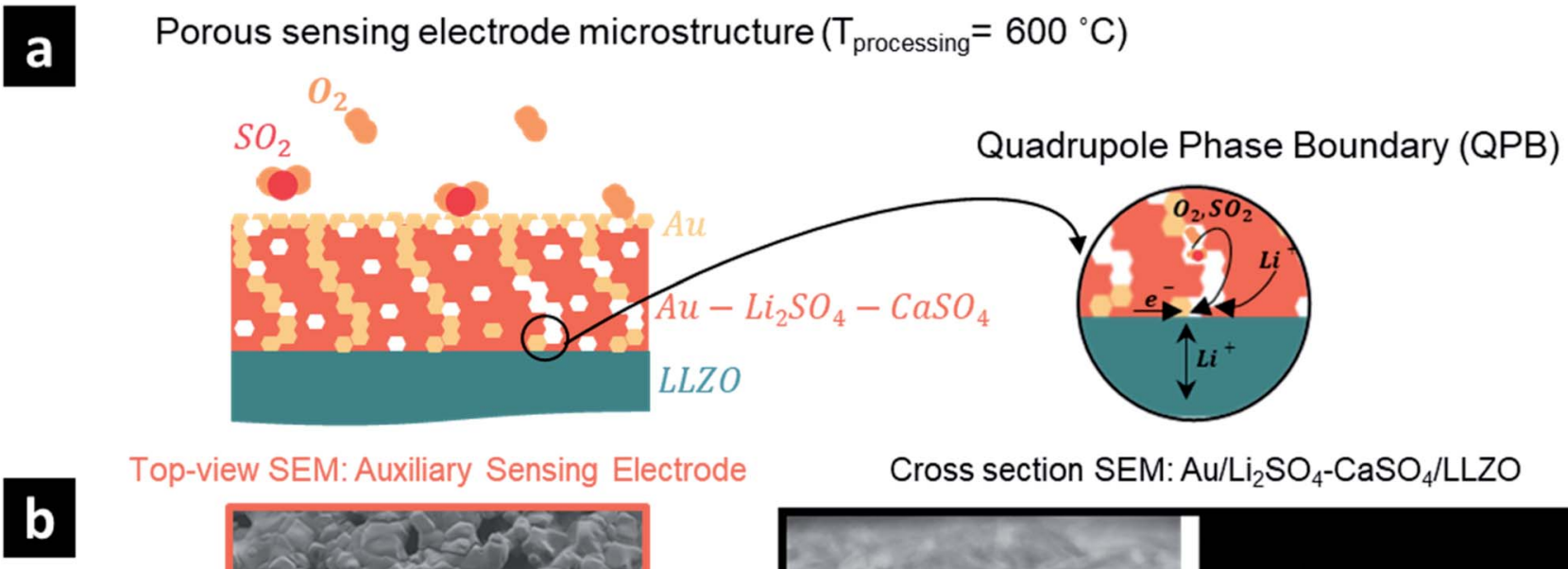

Top-view SEM: Auxiliary Sensing Electrode

Cross section SEM: Au/Li $\mathrm{SO}_{4}-\mathrm{CaSO}_{4} / \mathrm{LLZO}$
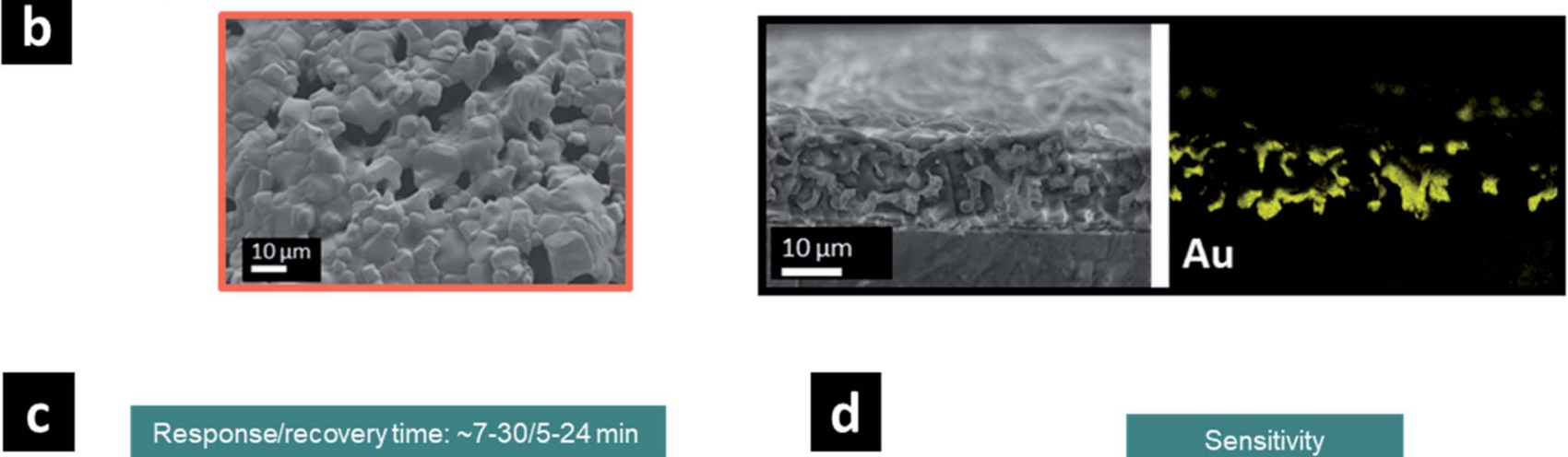

Response/recovery time: 7-30/5-24 min
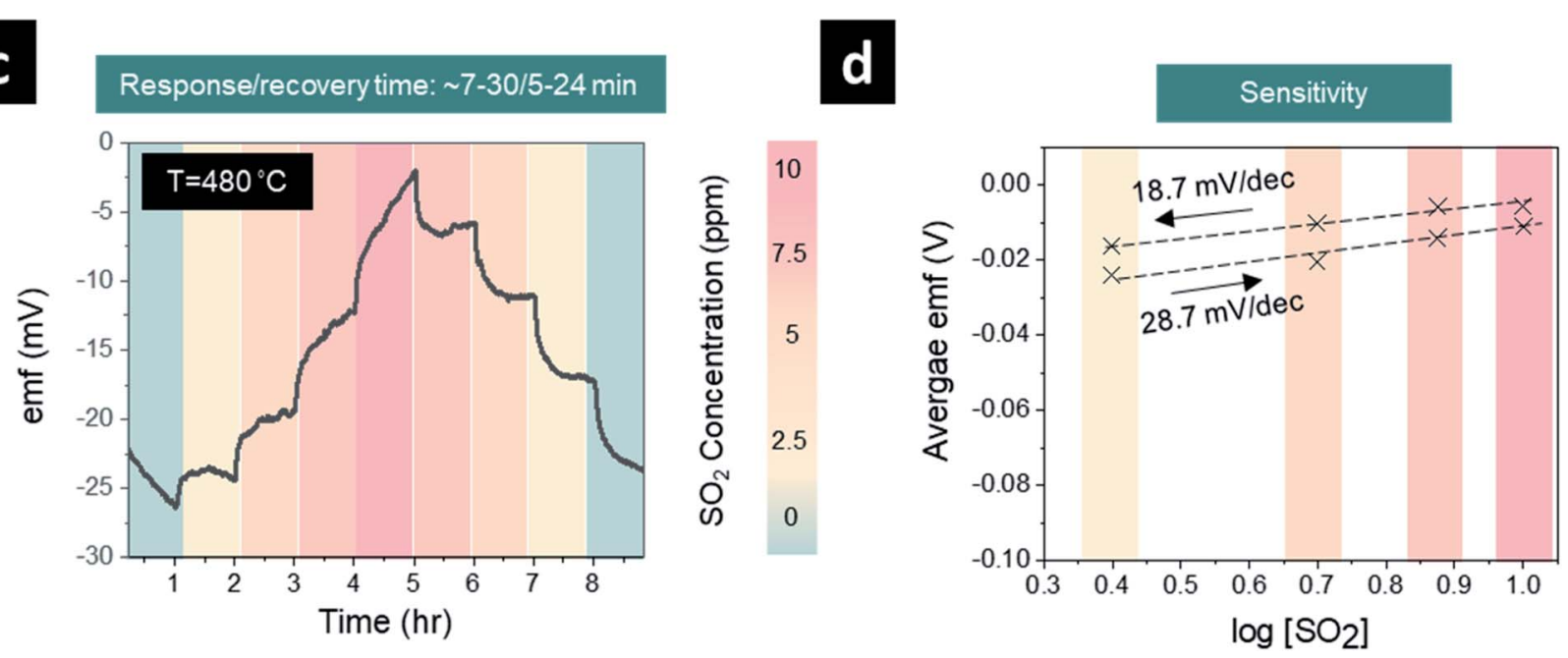

Fig. 3 (a) Schematic illustration of the $\mathrm{SO}_{2}$ sensor with a porous sensing electrode microstructure. (b) Top view and cross-sectional SEM micrographs and elemental analysis ( $\mathrm{Au}$ ) of dense auxiliary sensing electrodes processed at $600^{\circ} \mathrm{C}$. (c) Emf response to $\mathrm{SO}_{2} \mathrm{concentration}$ step change $(0-10 \mathrm{ppm})$ at $480^{\circ} \mathrm{C}$ of the porous auxiliary sensing electrodes (recovery/response times are stated above and were extracted from the graphs). (d) Emf dependence on the $\mathrm{SO}_{2}$ concentration on a logarithmic scale showing sensitivity for the porous auxiliary sensing electrodes.

phase of $\mathrm{Li}_{2} \mathrm{SO}_{4}-\mathrm{CaSO}_{4}$, the $\mathrm{Au}$ current collector, the LLZO electrolyte, and the gaseous phase of $\mathrm{SO}_{2}$ (see the schematic Fig. 3). The role of the auxiliary sensing electrode composition, microstructure, and interfacial reactions must be explored for QPB designs, and further attention regarding the optimum available surface area for the reaction is needed to ensure an adequate gas diffusion pathway and efficient adsorption/ desorption processes. In that sense, we propose two configurations of the $\mathrm{Li}_{2} \mathrm{SO}_{4}-\mathrm{CaSO}_{4}$ sensing electrode (one dense and one porous structure) by merely changing the processing temperature to ensure a larger effective area for the reaction at the QPB and explore the sensing characteristics toward $\mathrm{SO}_{2}$.

\section{Experimental}

\subsection{Synthesis of the LLZO solid electrolyte}

The solid electrolyte $\mathrm{Li}_{6.5} \mathrm{La}_{3} \mathrm{Zr}_{1.5} \mathrm{Ta}_{0.5} \mathrm{O}_{12}$ (LLZO) was prepared via the solid-state reaction route detailed elsewhere. ${ }^{13}$ In short, stoichiometric amounts of $\mathrm{La}(\mathrm{OH})_{3}$ (Sigma Aldrich, 99.9\%), $\mathrm{ZrO}_{2}$ (Sigma Aldrich, 99.9\%), and $\mathrm{Ta}_{2} \mathrm{O}_{5}$ (Sigma Aldrich, 99.99\%) and an excess amount of $50 \mathrm{wt} \% \mathrm{LiOH}$ (Alfa Aesar, purity $99.8 \%$ ) to compensate for the lithium evaporation during the high-temperature calcination of the powder were ball-milled in absolute isopropanol, dried, and calcined two times at $750^{\circ} \mathrm{C}$ for $10 \mathrm{~h}$ at a heating rate of $5{ }^{\circ} \mathrm{C} \mathrm{min}^{-1}$ under a constant flow of 
synthetic air. Finally, a weighed amount of the LLZO powder was pressed into a pellet using a die with a diameter of $12 \mathrm{~mm}$ and thickness of $1.5 \mathrm{~mm}$ in a uniaxial press (2.2 tons per $\mathrm{cm}^{2}$ ). The green pellets were sintered in a $\mathrm{MgO}$ crucible at $1100{ }^{\circ} \mathrm{C}$ for $5 \mathrm{~h}$ at heating/cooling rates of $10{ }^{\circ} \mathrm{C} \mathrm{min}^{-1}$ under a constant flow of pure oxygen $(50 \mathrm{sccm})$. The sintered LLZO pellets were dry-polished to ensure consistency among all the samples.

\subsection{Fabrication of the sensing electrode and sensor device}

Dense auxiliary sensing electrode. The auxiliary electrode was prepared by mixing different mole ratios of lithium sulfate ( $\mathrm{Li}_{2} \mathrm{SO}_{4}$; anhydrous, 99.99\% trace metal basis, BeanTown Chemical) and calcium sulfate $\left(\mathrm{CaSO}_{4}\right.$; anhydrous, $99.99 \%$ trace metal basis, BeanTown Chemical). A binder solution (a mixture of $\alpha$-terpineol and ethylene cellulose) was added at a weight ratio of $1: 2$ to the different $\mathrm{Li}_{2} \mathrm{SO}_{4}: \mathrm{CaSO}_{4}$ powder mixtures and was crushed and homogenized using a mortar until a smooth paste was produced. The paste was brushed on a quarter of the solid electrolyte and heated at $750{ }^{\circ} \mathrm{C}$ for $2 \mathrm{~h}$ at a heating rate of $10{ }^{\circ} \mathrm{C} \mathrm{min}^{-1}$ under a constant flow of pure oxygen. After the auxiliary (sensing) electrode preparation, gold paste (conductive epoxy GOLD paste, EMS) was brush-painted on the second quarter of the solid-electrolyte surface, serving as the reference electrode (RE). A thin layer of the gold paste was brushed on the auxiliary (sensing) electrode, serving as a current collector. Platinum wires $(0.1 \mathrm{~mm}$ diameter, $99.995 \%$, BeanTown Chemical) were connected to the auxiliary (sensing) and reference electrodes using the gold paste. The complete sensor construction was annealed at $300{ }^{\circ} \mathrm{C}$ for $4 \mathrm{~h}$ in an oxygen atmosphere to cure the gold paste. An adhesive sealant was applied on the reference electrode (898FS, Cotronics Corp.) and cured again at $300{ }^{\circ} \mathrm{C}$ for $2 \mathrm{~h}$ in an oxygen atmosphere.

Porous auxiliary sensing electrode. To prepare a porous auxiliary electrode, the auxiliary electrode processing temperature was reduced to $600{ }^{\circ} \mathrm{C}$ (instead of $750{ }^{\circ} \mathrm{C}$ ), while keeping all the other parameters the same.

\subsection{Sensing system setup and electrochemical sensing measurements}

The $\mathrm{SO}_{2}$ gas sensing experiments were conducted using a Linkam stage (HFS600E) with an internal volume of $\sim 50 \mathrm{~cm}^{3}$ equipped with a heating element in the temperature range of 25-600 ${ }^{\circ} \mathrm{C}$. The experimental set-up has been detailed elsewhere. ${ }^{13}$ In short, two automated mass flow controllers (red-y, Vogtlin instruments, Switzerland) were used to balance between 50 ppm $\mathrm{SO}_{2}$ (in dry synthetic air) and dry synthetic air (21\% volume $\mathrm{O}_{2}$ in $\mathrm{N}_{2}$ ) to achieve different concentrations of the analyzed gas, namely $0-10 \mathrm{ppm} \mathrm{SO}_{2}$ in $2.5 \mathrm{ppm}$ steps, held for 1-2 h. The open-circuit voltage (OCV) was measured using a Keithley 2612B electrometer. The reference and auxiliary (sensing) electrodes were connected using Pt wires, which were glued to the electrodes with gold paste. The sensitivity of the sensor was evaluated through sensing experiments, where the OCV was measured as a function of $\mathrm{SO}_{2}$ concentration at a constant and calibrated temperature of either $240,320,400$, or $480{ }^{\circ} \mathrm{C}$ at a heating/cooling rate of $10{ }^{\circ} \mathrm{C} \mathrm{min}^{-1}$.

\subsection{Surface and bulk characterization}

Scanning electron microscopy. Field-emission scanning electron microscopes (Ultra Plus and Supra55VP equipped with an energy-dispersive X-ray spectrometer for elemental analysis and mapping, Zeiss) and a scanning electron microscope (JEOL 7900F) equipped with a wide variety of detectors including an energy-dispersive X-ray spectrometer and soft X-ray emission spectrometer allowed efficient and parallel collection of very low-energy rays with chemical state analysis. The different scanning electron microscopes were used to characterize the microstructure of the sensing electrodes as a function of their processing procedures.

Differential scanning calorimetry. The melting temperature of the sensing electrode as a function of its composition was determined via differential scanning calorimetry (DSC)/ thermogravimetric analysis (TGA) of different mole ratios of $\mathrm{Li}_{2} \mathrm{SO}_{4}: \mathrm{CaSO}_{4}$, namely $1: 0,1: 1,4: 1,2: 1,1: 2$, and $4: 1$. The measurement was conducted under synthetic air from room temperature to $900{ }^{\circ} \mathrm{C}$, held for $5 \mathrm{~min}$, and cooled back to room temperature at a ramp rate of $10{ }^{\circ} \mathrm{C} \mathrm{min}^{-1}$ for both the heating and cooling steps.

\section{Results and discussion}

\subsection{Phase compatibility and synthesis protocol for $\mathrm{a} \mathrm{SO}_{2}$ sensing electrode/Li-garnet electrolyte joint}

As a first step, we had to define a processing protocol for the manufacture of a sensing electrode, where we could vary the pores and two-phase constituents via melting and firing on a pre-made sintered pellet. This was an important step, as it later defined the variability of the sensor electrode microstructures and bonding to LLZO and was critical for defining the QPBs and the tool to control the sensor performance for this interface. We designed and fabricated a sulfur dioxide sensor architecture in an in-plane geometry consisting of a Ta-doped $\mathrm{Li}_{7} \mathrm{La}_{3} \mathrm{Zr}_{2} \mathrm{O}_{12}$ (LLZO) pellet electrolyte with a sensing electrode and gold reference electrode forming a type III-electrochemical cell, Fig. 1a. For this, the gold reference electrode was brushpainted as a paste on top of the dense LLZO pellet and sealed with inorganic adhesive, serving as a protective layer for tight isolation from possible water vapor and $\mathrm{SO}_{2}$ gas in the atmosphere (Fig. 1b). One challenging aspect in the $\mathrm{SO}_{2}$ sensor design was to define the chemical composition of the sensing electrode, as it can largely determine the cell voltage and response of the sensor. It has been established that $\mathrm{Li}_{2} \mathrm{SO}_{4}$ can effectively serve as an auxiliary sensing electrode with a relatively fast response to $\mathrm{SO}_{2}$. However, sluggish recovery times necessitated two or multiple auxiliary phase components, with $\mathrm{CaSO}_{4},{ }^{7} \mathrm{BaSO}_{4},{ }^{6} \mathrm{~V}_{2} \mathrm{O}_{5},{ }^{17}$ or $\mathrm{La}_{2} \mathrm{O}_{2} \mathrm{SO}_{4}-\mathrm{K}_{2} \mathrm{SO}_{4}$ typically added to facilitate higher ionic conductivities and suppress interference attributed to the presence of humidity. ${ }^{18-21}$ For example, it was reported that by merely changing the chemical composition of the auxiliary sensing electrode from the single component $\mathrm{Li}_{2} \mathrm{SO}_{4}$ to multi-component $\mathrm{Li}_{2} \mathrm{SO}_{4}-\mathrm{CaSO}_{4}-\mathrm{SiO}_{2}$ at a molar ratio of $2: 2: 1$, a one-order-of-magnitude decrease in the average response $(\sim 15 \mathrm{~s})$ and recovery $(\sim 8 \mathrm{~min})$ rates to $\mathrm{SO}_{2}$ at $650{ }^{\circ} \mathrm{C}$ 
was observed when MgO-stabilized zirconia was used as the solid electrolyte. ${ }^{7}$ In the current study, the use of the Li-garnet solid electrolyte obviates the use of $\mathrm{SiO}_{2}$ to avoid the decomposition of the electrolyte through the formation of lithium silicate $\left(\mathrm{Li}_{2} \mathrm{Si}_{x} \mathrm{O}_{2 x+1}\right)$ compounds, which resulted in our selection of a two-component auxiliary sensing electrode, i.e. $\mathrm{Li}_{2} \mathrm{SO}_{4}-$ $\mathrm{CaSO}_{4}$. While creating the sensor, it was important to ensure good adhesion between the molten-salt-brushed and fired sensing electrode and the previously sintered pellet electrolyte. We identified an optimum 1 : 1 mole ratio of $\mathrm{Li}_{2} \mathrm{SO}_{4}-\mathrm{CaSO}_{4}$ (see ESI, Fig. S1, $\uparrow$ for further mixtures) for firing at $750{ }^{\circ} \mathrm{C}$ to ensure improved adhesion between the dense sensing electrode and solid electrolyte (Fig. 1c).

Collectively, the $\mathrm{SO}_{2}$ sensor was composed of the following electrochemical cell:

$$
\mathrm{Au}\left|\mathrm{Li}_{6.54} \mathrm{La}_{3.00} \mathrm{Zr}_{1.36} \mathrm{Ta}_{0.50} \mathrm{O}_{11.73}\right| \mathrm{Li}_{2} \mathrm{SO}_{4}-\mathrm{CaSO}_{4} \mid \mathrm{Au}, \mathrm{SO}_{2}, \mathrm{O}_{2} \text {, }
$$

where gold $(\mathrm{Au})$ is the reference electrode, $\mathrm{Li}_{6.54} \mathrm{La}_{3.00} \mathrm{Zr}_{1.36^{-}}$ $\mathrm{Ta}_{0.50} \mathrm{O}_{11.73}$ is the solid electrolyte, and $\mathrm{Li}_{2} \mathrm{SO}_{4}-\mathrm{CaSO}_{4}$ represents the binary compound of the auxiliary sensing electrode. When the cell was heated to stable thermal operation conditions, mobile $\mathrm{Li}^{+}$ions in the sensing electrode and solid electrolyte participated in the electrochemical reaction, and the cell reaction could reach an equilibrium state. The oxidation of $\mathrm{SO}_{2}$ at the auxiliary sensing electrode proceeds according to the following reaction:

$$
\mathrm{SO}_{2}+\frac{1}{2} \mathrm{O}_{2} \leftrightarrow \mathrm{SO}_{3}
$$

Thus, the overall reaction at the sensing electrode is

$$
2 \mathrm{Li}^{+}+\mathrm{SO}_{3}+\frac{1}{2} \mathrm{O}_{2}+2 \mathrm{e}^{-} \leftrightarrow \mathrm{Li}_{2} \mathrm{SO}_{4}
$$

At the reference electrode, $\mathrm{Li}^{+}$ions are expected to react mainly with oxygen (close reference configuration), possibly according to the following equation:

$$
2 \mathrm{Li}^{+}+\frac{1}{2} \mathrm{O}_{2}+2 \mathrm{e}^{-} \leftrightarrow \mathrm{Li}_{2} \mathrm{O}
$$

Under the equilibrium state, the emf of the sensor can be expressed according to the Nernst equation (Fig. 1d). Thus, under a fixed temperature and the partial pressure of $\mathrm{O}_{2}\left(p\left[\mathrm{O}_{2}\right]\right)$, the electromotive force, $E$, is determined by the partial pressure of $\mathrm{SO}_{2}\left(p\left[\mathrm{SO}_{2}\right]\right)$ and according to the Nernst equation:

$$
E=E^{0}+\frac{R T}{2 F} \ln \left(p\left[\mathrm{SO}_{2}\right]\right),
$$

where $E^{0}$ is the standard potential (constant), $F$ is the Faraday constant, $R$ the gas constant, and $T$ is the absolute temperature (K).

\subsection{Sensing electrode microstructure and $\mathrm{SO}_{2}$ sensor voltage response}

After elucidating the optimum protocol to design dense $\mathrm{Li}_{2} \mathrm{SO}_{4}-$ $\mathrm{CaSO}_{4}$ sensing electrodes, we investigated the effect of having either a TPB (Fig. 2) or QPB (Fig. 3) for the electrochemical reaction sites, whereby first a dense structure and second a porous structure were achieved, respectively, by manipulating the sensing electrode firing temperatures from $750{ }^{\circ} \mathrm{C}$ (Fig. 2) to $600{ }^{\circ} \mathrm{C}$ (Fig. 2), respectively. In the following section, the full sensor design and testing are reported.

With the material design and theoretical concept for $\mathrm{SO}_{2}$ sensing, we then targeted the $\mathrm{SO}_{2}$ sensor operation to investigate its performance characteristics employing a dense sensing electrode microstructure (Fig. 2a and b). We set the sensing protocol by modulating the $\mathrm{SO}_{2}$ concentration from 0 to $10 \mathrm{ppm}$ and down to 0 ppm with $2.5 \mathrm{ppm}$ step changes in dry synthetic air. The electromotive force (emf) values were recorded to define the $\mathrm{SO}_{2}$ concentration sensitivity in detection; the response and recovery times were also recorded. Note that these times were defined as the time required for the sensor to reach $90 \%$ of the steady final signal and for the sensor to return to $10 \%$ of the steady signal, respectively.

The emf response of the electrochemical cell to the $\mathrm{SO}_{2}$ concentration steps at $480{ }^{\circ} \mathrm{C}$ is depicted in Fig. 2c. During the concentration step change of $\mathrm{SO}_{2}$ from 0 to $10 \mathrm{ppm}$, the emf voltage response implies a difference in the chemical potentials of $\mathrm{Li}^{+}$ions in the garnet LLZO solid electrolyte established at the interfaces with the sensing and reference electrodes, respectively. Moreover, the initial negative polarity in the presence of synthetic air or low $\mathrm{SO}_{2}$ concentrations indicates an accumulation of $\mathrm{Li}^{+}$ions at the reference electrode and the depletion of $\mathrm{Li}^{+}$ions at the sensing electrode. The transfer of $\mathrm{Li}^{+}$ions is accompanied by the occurrence of reaction [2] from right to left at the sensing electrode interface and reaction [3] from left to right at the $\mathrm{Au}$ reference electrode. Increasing the $\mathrm{SO}_{2}$ concentration further induced the reverse transfer of $\mathrm{Li}^{+}$ions from the reference electrode to the sensing electrode and the opposite occurrence of reactions [2] and [3]. ${ }^{20}$ The average emf values and the logarithm of the $\mathrm{SO}_{2}$ gas concentration were fitted with the Nernst equation to evaluate the sensitivity of the sensor and were determined to be $144\left(n=1.3 \mathrm{e}^{-}\right)$and $76\left(n=1.96 \mathrm{e}^{-}\right) \mathrm{mV}$ $\mathrm{dec}^{-1}$ for the sensor response step of 0-10 ppm and a recovery step of 10-0 ppm (Fig. 1d). These values are similar to or higher than the theoretical sensitivity for a two-electron reaction of $74.64 \mathrm{mV} \mathrm{dec}{ }^{-1}$. The differences between the theoretical and calculated sensitivity, especially in the response stage, imply more efficient desorption, compared to adsorption, of $\mathrm{SO}_{2}$ gas at the surface of the sensing electrode. It is evident that the recovery appears easier to achieve within $\sim 15-60$ min depending on the $\mathrm{SO}_{2}$ concentration, whereas the response time was typically longer than $>60 \mathrm{~min}$ (except in the case of $0-2.5 \mathrm{ppm}$, where $t_{90 \%}=21 \mathrm{~min}$ ). Although the emf in the recovery stage was stable, it did not return to the initial emf value, and we conclude that a longer period beyond $>2 \mathrm{~h}$ may be required to ensure full recovery. The long response and recovery times may be attributed to the lack of a catalyst for fast $\mathrm{SO}_{2}$ oxidation and long processes of adsorption and desorption of $\mathrm{SO}_{2}$ in addition to the TPB-based sensing electrode dense design, which can significantly affect the sensing capabilities.

One promising strategy to shorten the response/recovery time is to introduce a porous auxiliary sensing electrode to 
facilitate faster adsorption and diffusion of sulfur dioxide through a "porous" sensing electrode structure (Fig. 3a and b). This strategy could increase the effective surface area for reaction and the number of sulfur dioxide molecules that participate in the electrochemical reaction. In general, the sensing reaction occurs at the QPB reactive sites in the case of a porous auxiliary sensing electrode (or at the TPB sites in the case of a dense electrode, Fig. 2a), where the electrochemical equilibrium requires a quadruple contact between the auxiliary phase of $\mathrm{Li}_{2} \mathrm{SO}_{4}-\mathrm{CaSO}_{4}$, the Au current collector, the LLZO electrolyte, and the gaseous phase of $\mathrm{SO}_{2}$ (Fig. 3a). To improve the distribution of $\mathrm{Au}$ particles and increase the density of QPB reaction sites throughout the sensing electrode, we designed in the next step a porous microstructure for the sensing electrode with an approximate pore diameter ranging from 5 to $10 \mu \mathrm{m}$ by lowering the processing temperature of the sensing electrode from 750 to $600{ }^{\circ} \mathrm{C}$ (Fig. 3b). Turning to a porous sensing electrode structure, we analyzed the response and recovery times at $480{ }^{\circ} \mathrm{C}$ to $2.5 \mathrm{ppm} \mathrm{SO}_{2}$ in the concentration ranges of $0-10 \mathrm{ppm}$ and $10-0$ ppm (Fig. 3c). The graph shows a clear improvement in the response time of the porous sensing electrode compared with the dense electrode processed at two different temperatures. Here, the porous sensing electrodes reduced the response time from $>\sim 60 \mathrm{~min}$ (dense) to $\sim 7-30 \mathrm{~min}$ (porous) and the recovery time from $\sim 15-60 \mathrm{~min}$ (dense) to $\sim 5-24 \mathrm{~min}$ (porous) depending on the selected $\mathrm{SO}_{2}$ concentration step within 0$10 \mathrm{ppm}$. The cross-sectional scanning electron microscopy (SEM) images and energy-dispersive X-ray spectroscopy (EDS) elemental mapping of the sensing electrode/solid electrolyte interface (Fig. 3b) revealed that the porous sensing electrode structure with an average thickness of $\sim 20 \mu \mathrm{m}$ ensured homogeneous incorporation of the gold paste into the porous sensing electrode, creating multiple QPB contact points for the cermet structure, which account for the improvement in the response
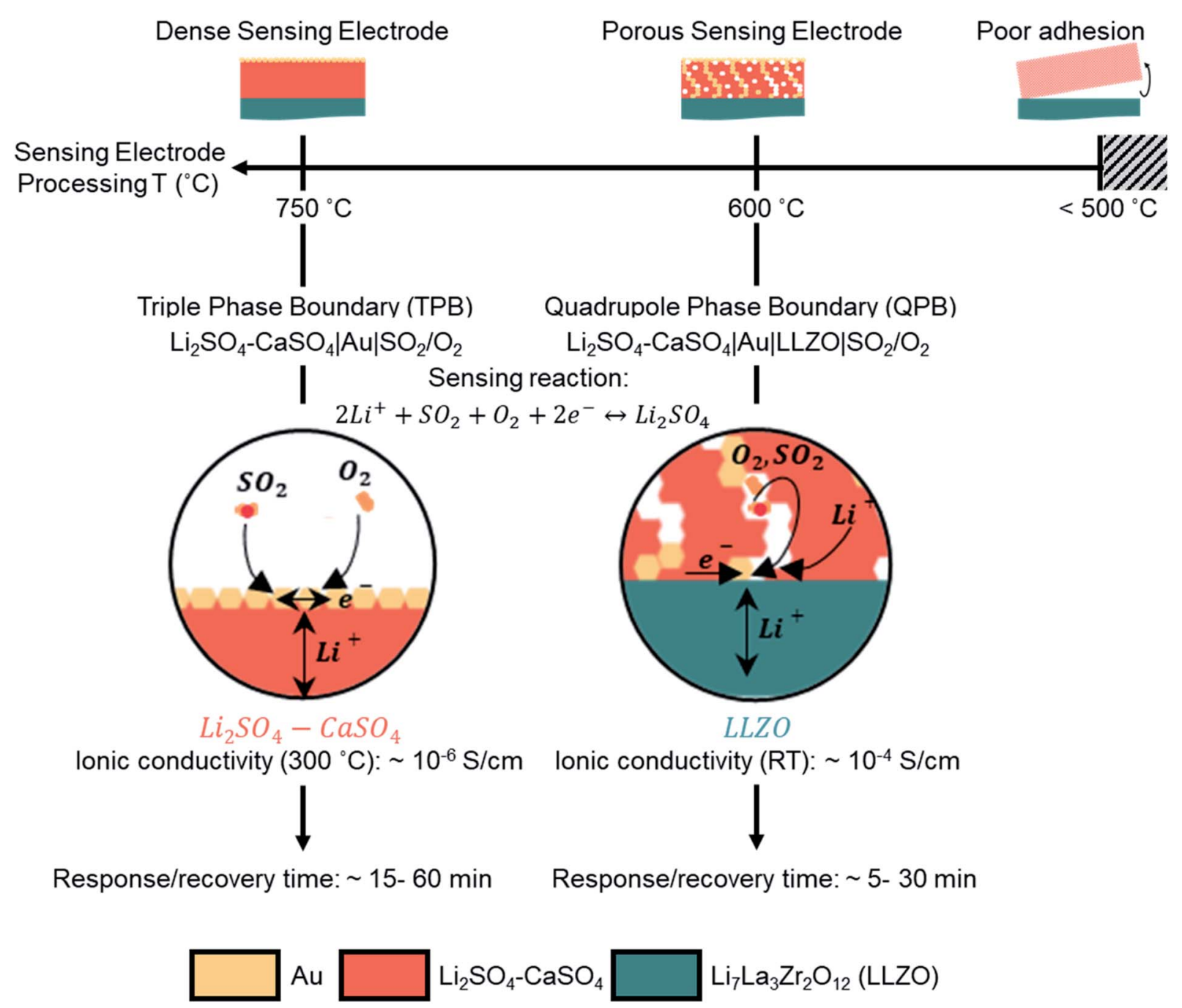

Fig. 4 Schematic illustration of the effect of the sensing electrode processing temperature on the microstructure and $\mathrm{SO}_{2}$ sensor performance characteristics, namely the response and recovery times. The manipulation of processing temperatures, either 750 or $600{ }^{\circ} \mathrm{C}$, resulted in a dense or porous microstructure of the sensing electrode, respectively, and shifted the electrochemical reaction from $\mathrm{TPB}\left(\mathrm{Li}_{2} \mathrm{SO}_{4}-\mathrm{CaSO}{ }_{4}, \mathrm{Au} \mathrm{current}\right.$ collector, and $\left.\mathrm{SO}_{2} / \mathrm{O}_{2}\right)$ to QPB $\left(\mathrm{Li}_{2} \mathrm{SO}_{4}-\mathrm{CaSO}_{4}\right.$, Au current collector, LLZO electrolyte, and $\left.\mathrm{SO}_{2} / \mathrm{O}_{2}\right)$ zones. In the QPB-based configuration, the diffusion pathways of $\mathrm{Li}^{+}$in the poor $\mathrm{Li}_{2} \mathrm{SO}_{4}-\mathrm{CaSO}_{4}$ conductor were significantly shorter than those in the TPB-based configuration. 
time after $\mathrm{SO}_{2}$ exposure (Fig. 3b). Although it is a common practice to mitigate the challenge of slow response times after gas exposure by incorporating gold nanoparticles into the composite sensing electrode to maintain better metallic conductivity and improve the phase boundary contact areas, this approach resulted in limited success in our case. Alternatively, our porous sensing electrode structure supported a continuous ion percolating network for $\mathrm{Li}_{2} \mathrm{SO}_{4}$ and LLZO phases and the electronic one for the gold particles accompanied by the infiltration of sulfur dioxide into the porous structure (Fig. 3b) and effectively increased the available surface area for the electrochemical reaction. Nonetheless, the fly in the ointment is that a porous structure with efficient QPBs may not only ensure a higher effective surface area for reaction and favorable diffusion of sulfur dioxide into the porous sensing electrode structure but may also induce additional degradation reactions between the solid electrolyte and sensing electrode. Indeed, once the average emf values and the logarithm of the $\mathrm{SO}_{2}$ gas concentration were fitted with the Nernst equation to evaluate the sensitivity $\left(\mathrm{mV} \mathrm{dec}^{-1}\right)$, the sensitivity was determined to be $28.7 \mathrm{mV} \mathrm{dec}^{-1}\left(n=5.2 \mathrm{e}^{-}\right)$and $18.7 \mathrm{mV} \mathrm{dec}^{-1}(n=$ $\left.7.9 \mathrm{e}^{-}\right)$for the sensor response step $(0-10 \mathrm{ppm})$ and recovery step $(10-0 \mathrm{ppm})$, respectively, significantly lower than the theoretical sensitivity of $74.64 \mathrm{mV} \mathrm{dec}^{-1}$ and the sensitivities determined for the dense sensing electrode (Fig. 3d). When the voltage does not approximately follow the Nernst law, the system is considered to be not in thermodynamic equilibrium. The higher electron number determined, compared to the theoretical one, may imply that additional degradation reactions are taking place and necessitate further investigation.

Our investigation revealed that by lowering the processing temperature of the $\mathrm{Li}_{2} \mathrm{SO}_{4}-\mathrm{CaSO}_{4}$ sensing electrode from 750 to $600{ }^{\circ} \mathrm{C}$, the transition from a dense to a porous structure of the sensing electrode, i.e. from TPBs to QPBs, can be easily achieved (Fig. 4). The importance of transitioning to a porous QPB-based configuration is two-fold: (i) the formation of the porous electrode structure increases the surface area for the electrochemical sensing reaction to occur, which may facilitate faster gas adsorption/desorption processes and improve the response/ recovery time and (ii) the $\mathrm{Li}^{+}$-ion diffusion pathways in the lowconducting $\mathrm{Li}_{2} \mathrm{SO}_{4}-\mathrm{CaSO}_{4}$ phase $\left(\sim 10^{-6} \mathrm{~S} \mathrm{~cm}^{-1}\right.$ at $\left.300{ }^{\circ} \mathrm{C}\right)$ are shortened compared to the $\mathrm{Li}^{+}$-ion diffusion pathways through dense, several-micron-thick sensing electrodes. The low sensitivities observed for the porous sensing electrode microstructure imply that the voltage does not approximately follow the Nernst law and that the system is considered to not be in thermodynamic equilibrium. The higher electron number determined, compared to the theoretical one, may imply that additional degradation reactions are occurring and necessitate further investigation.

\section{Conclusion}

Successful design of an electrochemical potentiometric type III sensor requires thoughtful selection of materials and microstructures that balance ion-electron-gas exchange processes at thermodynamically stable materials and interfaces under a corrosive $\mathrm{SO}_{2}$ environment. In this work, we explored electrochemical sensing electrode designs concerning their ideal chemistry and ion-electronic pathways toward a Li-garnet, $\mathrm{Li}_{6.54} \mathrm{La}_{3.00} \mathrm{Zr}_{1.36} \mathrm{Ta}_{0.50} \mathrm{O}_{11.73}$, solid electrolyte for potentiometric type III $\mathrm{SO}_{2}$ sensors. Only very recently, a first proof-of-concept was demonstrated for the suitability of a well-known battery electrolyte for $\mathrm{SO}_{2}$ sensors; however, guidelines on how to design the electrode microstructure and chemistry toward fast ionic transfer have remained untouched. A main requirement for the functionality of this sensor is the assurance of fast transport kinetics in the ionic, electrical, and gas boundary zones. A challenging part of this electrochemical device is that multiple phases of ion conductors $\left(\mathrm{Li}_{2} \mathrm{SO}_{4}-\mathrm{CaSO}_{4}\right)$ and electronic conductors (gold) must be used to ensure ionic-electronic pathways, while also allowing enough gas exchange with $\mathrm{SO}_{2}$ for adsorption and desorption. For this, we created model sensing electrode structures composed of $\mathrm{Li}_{2} \mathrm{SO}_{4}-\mathrm{CaOS}_{4}$ and defined as either dense or porous and tested the cells with a porous gold layer as the reference electrode. To succeed in defining the $\mathrm{SO}_{2}$ sensing electrode chemistry and phase and its stability toward the new LLZO electrolyte, we had to probe the phase compatibility and invent a synthesis protocol for the $\mathrm{SO}_{2}$ sensing electrode/electrolyte joint under the critical and societyrelevant limit of $0-10 \mathrm{ppm} \mathrm{SO}_{2}$ at $480{ }^{\circ} \mathrm{C}$. The focus was strategically placed on one of the major aspects, which affects the emf response according to the Nernstian behavior and the response/recovery time of the sensor, explicitly the microstructure of the auxiliary sensing electrode.

We discovered that it is possible by ceramic design to ensure a larger effective area for the $\mathrm{SO}_{2}$ sensing reaction and two configurations were presented, dense and porous structures of the $\mathrm{Li}_{2} \mathrm{SO}_{4}-\mathrm{CaSO}_{4}$ sensing electrode, by merely lowering the processing temperature. The last manipulation enforced the desired shift from TPB to QPB reaction zones, as pores were formed and unlocked new effective electrochemical sensing zones with conductive Au channels. We identified that the QPBbased configuration resulted in up to a one-order-of-magnitude lowered response time and up to $75 \%$ decrease in the recovery time down to $\sim 5$ min compared with the TPB-based configuration and was superior in performance. This work contributes to the first discussions in the field on how such catalyst-free sensor electrode designs could look like and provides the first performance characteristics and manufacturing guidelines. We conclude that the electrochemical concept of QPBs clearly shows potential and can be further extended considering other auxiliary phases for $\mathrm{SO}_{2}$ sensors or may be considered for other type III garnet-based sensors for future $\mathrm{CO}_{2}, \mathrm{NO}_{2}$, and $\mathrm{H}_{2} \mathrm{~S}$ tracking as a strategy to design structure-to-performance for their sensing electrodes.

\section{Conflicts of interest}

The authors declare no conflicts of interest.

\section{Acknowledgements}

This work was supported in part by SENSE.nano under award number 2629303. M. B. acknowledges financial support from 
the US-Israel Fulbright Program, the Zuckerman Israeli Postdoctoral Scholar Program, and the MIT-Technion Postdoctoral Fellowship. J. L. M. R. thanks the Thomas Lord Foundation for financial support. This work made use of the MRSEC Shared Experimental Facilities at MIT, supported by the National Science Foundation under award number DMR-1419807. This work was performed in part at the Center for Nanoscale Systems (CNS), a member of the National Nanotechnology Coordinated Infrastructure Network (NNCI), supported by the National Science Foundation under NSF award no. 1541959. CNS is part of Harvard University.

\section{References}

1 S. Dahiya, A. Anhäuser, A. Farrow, H. Thieriot, A. Kumar and L. Myllyvirta, Ranking the World's Sulfur Dioxide $\left(\mathrm{SO}_{2}\right)$ Hotspots: 2019-2020 A closer look at the colourless gas that is poisoning our air and health, 2020.

2 Q. Zhong, H. Shen, X. Yun, Y. Chen, Y. Chen, Y. Ren, H. Xu, G. Shen, W. Du, W. Du, J. Meng, W. Li, J. Ma and S. Tao, Environ. Sci. Technol., 2020, 54, 6508-6517.

3 L. Cubic Sensor and Instrument Co., Online Infrared Flue Gas Analyzer-Gasboard 3000, https://en.gassensor.com.cn/ GasAnalyzer/info_itemid_291.html, accessed 20 January 2021.

4 Acoem-ECOTECH, The Serinus ${ }^{\circledR} 50$ sulfur dioxide (SO2) analyser, 2020.

5 Thermo Scientific, User Manual: 43iQ Sulfur Dioxide Analyzer, 2020.

6 Y. Yan, Y. Shimizu, N. Miura and N. Yamazoe, Chem. Lett., 1992, 21, 635-638.

7 Y. Yan, Y. Shimizu, N. Miura and N. Yamazoe, Sens. Actuators, B, 1994, 20, 81-87.
8 R. Akila and K. T. Jacob, Sens. Actuators, 1989, 16, 311-323. 9 S.-D. Choi, W.-Y. Chung and D.-D. Lee, Sens. Actuators, B, 1996, 36, 263-266.

10 K. J. Kim, M. Balaish, M. Wadaguchi, L. Kong and J. L. M. Rupp, Adv. Energy Mater., 2020, 2002689.

11 M. Balaish, J. C. Gonzalez-Rosillo, K. J. Kim, Y. Zhu, J. D. Hood and J. L. M. Rupp, Nat. Energy, 2021, 6, 227-239.

12 Y. Zhu, J. C. Gonzalez-Rosillo, M. Balaish, Z. D. Hood, K. J. Kim and J. L. M. Rupp, Nat. Rev. Mater., 2021, 6, 313331.

13 M. Balaish and J. L. M. Rupp, Adv. Mater., 2021, 33(20), 2100314.

14 M. Struzik, I. Garbayo, R. Pfenninger and J. L. M. Rupp, Adv. Mater., 2018, 30, 1-10.

15 J. C. Gonzalez-Rosillo, M. Balaish, Z. D. Hood, N. Nadkarni, D. Fraggedakis, K. J. Kim, K. M. Mullin, R. Pfenninger, M. Z. Bazant and J. L. M. Rupp, Adv. Mater., 2020, 32, 1907465.

16 M. Balaish, Z. D. Hood and J. L. M. Rupp, Gas sensor device containing Lithium garnet, US Pat., 63, 2021.

17 K. Chen, M. Zhang, H. Wang and H. Gu, Rev. Sci. Instrum., 2019, 90, 065001.

18 G. Hötzel and W. Weppner, Sens. Actuators, 1987, 12, 449453.

19 A. Lundén, K. Schroeder and H. Ljungmark, Solid State Ionics, 1988, 28-30, 262-266.

20 F. Ménil, B. O. Daddah, P. Tardy, H. Debéda and C. Lucat, Sens. Actuators, B, 2005, 107, 695-707.

21 K. Singh and V. K. Deshpande, Solid State Ionics, 1984, 13, 157-164. 